\title{
Determinación de acrilamida en el procesamiento de la panela por cromatografía líquida
}

\author{
Acrylamide Determintion in the Sugar Cane Juice Process by the Liquid \\ Chromatography Technique
}

\author{
J. J. Vargas Lasso ${ }^{\mathrm{a}, *}$ \\ Y. V. Talero Pérez ${ }^{\mathrm{b}}$ \\ F. A. Trujillo Suárez ${ }^{\mathrm{c}}$ \\ L. R. Camelo Caballero ${ }^{\mathrm{d}}$
}

Recepción: 12-oct-14

Aceptación: 25-nov-14

\section{Resumen}

Se evaluó una nueva metodología para detectar y cuantificar la formación de acrilamida en las etapas de producción de la panela. Los datos de esta investigación contribuyen al control de calidad de la panela, particularmente en la evaluación del riesgo químico asociado a este producto de uso popular, en beneficio primordial del sector agroalimentario y de la calidad. La técnica de análisis empleada fue la cromatografía líquida de alta eficiencia en fase reversa, y el método de cuantificación empleado fue el estándar externo, para lo cual se construyó una curva de calibración. Se halló que en el jugo de caña no se forma acrilamida, pero a medida que aumenta el tiempo y la temperatura (reacción de Maillard) se evidencia su presencia,encontrándose en el producto final $2.0 \mathrm{mg} / \mathrm{kg}$; se pudo, en consecuencia, establecer la efectividad de la cuantificación por este método analítico; para la verificación y confirmación del contenido de acrilamida, información útil para evaluar la autenticidad del producto final.

Palabras clave: Acrilamida, Asparagina, Azúcares reductores, HPLC, Jugo caña de azúcar, Reacción de Maillard.

\begin{abstract}
A new method for the detection and quantification of acrylamide formation in the production stages of sugar cane was evaluated. This research provides data to help the food industry, particularly in quality control of sugar cane, for chemical risk evaluation associated with this popular product and often used for primary
\end{abstract}

\footnotetext{
${ }^{a}$ Facultad de Ciencias, Universidad Pedagógica y Tecnológica de Colombia (UPTC) Tunja, Grupo de investigación química y tecnología de alimentos, GIQTA.

*Autor de correspondencia: john.vargaslasso@uptc.edu.co

${ }^{\text {b} P o n t i f i c i a ~ U n i v e r s i d a d ~ J a v e r i a n a, ~ Q u i ́ m i c o ~ d e ~ a l i m e n t o s ~ U n i v e r s i d a d ~ P e d a g o ́ g i c a ~ y ~ T e c n o l o ́ g i c a ~ d e ~ C o l o m b i a ~(U P T C) ~ T u n j a . ~}$

${ }^{c}$ Fundación Universitaria Internacional del Trópico Americano - Unitrópico, Facultad de Ingeniería, Programa de Ingeniería de Alimentos, sede Yopal. Grupo de Investigación en ciencias Básicas Aplicación e innovación - CIBAIN.

${ }^{\mathrm{d}}$ Universidad de Buenos aires (UBA), Químico de alimentos Universidad Pedagógica y Tecnológica de Colombia (UPTC), Tunja.
} 
benefit of the quality and food industry. The technique employed was high efficiency liquid chromatography in reverse phase, the quantification method employed was the external standard, for which a calibration curve was constructed. Getting in cane juice, acrylamide formation does not occur, but with increasing time and temperature (Maillard reaction) its presence is evident; found in the final product $2.0 \mathrm{mg} / \mathrm{kg}$, so it can establish the effectiveness of quantification for this analytical method, for verification and confirmation of acrylamide, also contribute useful for evaluation of the authenticity of the final product information.

Key words: Acrylamide, Asparagine, Reducing Sugars, HPLC, Sugar Cane Juice, Maillard Reaction.

\section{Introducción}

El procesado de alimentos se puede considerar como el conjunto de prácticas que utilizan tecnologías y técnicas para trasformar los alimentos crudos, o productos intermedios, en alimentos listos para el consumo [1]. A través del tiempo, se han empleado numerosos métodos de preservación de los alimentos y de mejoramiento de su sabor, como la salazón y el secado, entre otros; y hoy es evidente que el procesamiento de los alimentos ha permitido avanzar en el abastecimiento de ellos, al prolongar su vida útil y aumentar la variedad de productos disponibles; sin embargo, estos hábitos alimenticios se han descentralizado, al dejar de cubrir la demanda nutricional (calidad y seguridad alimentaria) y pasar a cubrir solamente las necesidades de supervivencia, sin importar la salud y bienestar de la comunidad.

Procesar alimentos por calor conduce a la formación de nuevos compuestos que inciden en la aceptabilidad del producto por el consumidor; mediante este proceso, los constituyentes de los alimentos experimentan una serie de reacciones, muchas de las cuales son responsables del aroma, el color, la textura y el sabor, pero otras están asociadas con la generación de compuestos potencialmente tóxicos [2]. En algunos casos, el empleo de altas temperaturas, en combinación con otros factores externos al alimento, da lugar a la formación de compuestos tóxicos que reducen el valor biológico e inciden en la seguridad de los alimentos; estas sustancias se denominan contaminantes químicos de procesado. Los contaminantes químicos de procesado son compuestos que no estaban presentes en el alimento fresco y cuya génesis está directamente relacionada con el proceso tecnológico o culinario aplicado. $\mathrm{La}$ mayoría de estos compuestos están relacionados con actividades mutagénicas, teratogénicas y carcinogénicas, entre otras, en organismos vivos, y por ello deben evaluarse y, si es necesario, ejercer las medidas de control necesarias por parte de las agencias de seguridad alimentaria estatales correspondientes [1]. De aquí nace la necesidad de estudiar y analizar estos procedimientos y de divulgar sus hallazgos y conclusiones, en unión con las entidades encargadas de la evaluación de riesgos y beneficios de los alimentos con respecto a la salud humana [3].

En consecuencia, el objetivo de este estudio fue investigar la presencia de acrilamida en las diferentes etapas del proceso de elaboración de la panela. La panela fue seleccionada debido a su popularidad y uso frecuente. El objetivo principal fue establecer una metodología confiable para la verificación del contenido elemental, así como contribuir con información útil para la evaluación de autenticidad del producto o adulteración. No es el alcance de este trabajo evaluar los posibles efectos toxicológicos producidos por el consumo de estos productos, ni estimar la ingesta diaria tolerable de acuerdo con las recomendaciones de los organismos de salud.

\section{Acrilamida}

La acrilamida (2-propenamida) es una molécula que contiene un doble enlace electrófilo y un grupo amida; se trata de una sustancia blanca e inodora, que a temperaturas superiores a $84,5{ }^{\circ} \mathrm{C}$ sufre polimerización espontánea [4]; es un compuesto hidrofílico de bajo peso molecular, y conocida principalmente por su uso como monómero en la producción de poliacrilamida [5]. La acrilamida y sus análogos han sido ampliamente utilizados en la industria de la química y el medioambiente $[4,5]$. Las acrilamidas también son un componente del humo del tabaco, que dio la primera indicación de que puede estar formado por calentamiento de material biológico. También se encontró en ciertos alimentos (fritos y asados)que contenían almidón y fueron procesadosa altas temperaturas (más de $120{ }^{\circ} \mathrm{C}$ ). Los niveles de acrilamida se incrementaron al aumentar el tiempo de calentamiento [6, 7]. Este compuesto, identificado hace tiempo como un peligro potencial industrial, ahora se ha encontrado en muchos alimentos [8]. 


\section{Panela}

La producción de panela es una de las agroindustrias de mayor tradición en los países tropicales. La panela, también denominada "chancaca" o "rapadura", en algunos países de América Latina, y "gur" o "Jaggery", en Asia, es un producto alimenticio que se obtiene del jugo de caña de azúcar (Saccharumofficinarum $L$.); se trata de un azúcar natural e integral y no centrifugado. Su presentación común al consumidor es en forma de bloques sólidos, aunque últimamente también se comercializa en forma granulada.

La panela se diferencia del azúcar blanco y rubio no solo en su apariencia física, sino en su composición química, pues contiene, además de sacarosa, glucosa y fructosa, diversos minerales tan importantes como calcio, fósforo, hierro, potasio y magnesio, entre otros [9], lo cual la hace mucho más rica nutricionalmente que el azúcar [10].

Según cifras de la FAO, 26 países en el mundo producen panela, y Colombia es el segundo productor después de la India, con un volumen que representa el 12,1\% de la producción mundial en el año 2001. Sin embargo, en términos de consumo por habitante, Colombia ocupa el primer lugar, con un consumo per cápita de $34,2 \mathrm{Kg} / \mathrm{año}$; este producto hace parte de la economía y canasta familiar de muchas de las familias colombianas [9].

El proceso de elaboración de panela consiste en moler la caña, clarificar y evaporar el jugo hasta obtener una miel concentrada (más de $90^{\circ}$ Brix), la cual se bate, moldea y enfría para lograr la solidificación. En las etapas de clarificación, encalado y punteo se emplea el calentamiento del jugo de caña, con el fin de concentrarlo y eliminar impurezas. La clarificación consiste en la eliminación de las cachazas o sólidos en suspensión, tales como bagacillos (material lignocelulósico), hojas, arenas, tierra, sustancias coloidales y sólidos solubles presentes en el jugo de la caña. La limpieza de los jugos ocurre gracias a la acción combinada del calentamiento suministrado por la hornilla y la acción de ciertos aglutinantes; estos se sumergen directamente en el jugo cuando se alcanzan temperaturas entre $50^{\circ}$ y $85^{\circ} \mathrm{C}$; a los 75 ${ }^{\circ} \mathrm{C}$ se retiran las impurezas denominadas "cachaza negra", y a los $95^{\circ} \mathrm{C}$ se retira la "cachaza blanca". El encalado es una parte de la limpieza que consiste en adicionarle cal (óxido de calcio) al jugo, con el fin de regular su acidez en un valor de $\mathrm{pH}$ de 5.8, para prevenir la formación de azúcares reductores y ayudar a la clarificación de los jugos; la aplicación se realiza en dos etapas: a $40{ }^{\circ} \mathrm{C}$ y $80^{\circ} \mathrm{C}$. El punteo corresponde a la etapa de evaporación y concentración del jugo de caña,a temperaturas entre $\operatorname{los} 98^{\circ} \mathrm{C}$ $\mathrm{y} \operatorname{los} 120^{\circ} \mathrm{C}[11,12]$.

\section{Metodología}

\subsection{Materia prima}

Las muestras se recogieron en envases ámbar de $250 \mathrm{~mL}$, del trapiche "Los molinos", ubicado en el municipio de Santana, en el departamento de Santander. Estas fueron almacenadas en una nevera portátil para ser trasladadas hasta el Laboratorio de investigación de química y tecnología de alimentos (GIQTA), de la UPTC; el muestreo se realizó en cada una de las etapas de elaboración a diferentes temperaturas; todas las muestras fueron procesadas por triplicado y mantenidas en las mismas condiciones durante todo el estudio.

\subsection{Instrumentación}

La extracción y tratamiento de las muestras se realizó con ayuda de una centrífuga Rotina 46s marca Hettythzentrifugen, una balanza analítica Adventure Ohaus,una estufa de secado Binder y un molino con cuchillas extrafinas marca Ika, y finalmente las muestras fueron analizadas en un equipo de cromatografía líquida de alta resolución con detector ultravioleta visible, marca Agilent Tecnologies serie S1200, con bomba cuaternaria, provisto de un sistema de muestra manual y un desgasificador incorporado; además, este equipo cuenta con el software EZChrome.

Reactivos. Los patrones y reactivos empleados fueron grado analítico, Acrilamida Aldrich Alder (99\% de pureza), Acetonitrilo (JT Baker), etanol (JT Baker), agua desionizada, los filtros fueron adquiridos por Merck, Colombia.

Preparación de la muestra. Las muestras se recolectan en diferentes etapas del proceso de elaboración de la panela (extracción, prelimpieza, clarificación y encalado, evaporación y concentración, punteo y batido), para posteriormente ser analizadas por HPLC, por lo cual se realiza el siguiente procedimiento: para las muestras líquidas se tomó $1 \mathrm{~mL}$ 
de jugo de caña y se disolvió en $100 \mathrm{~mL}$ de agua desionizada y filtrada. En cuanto a las muestras sólidas, se pesó $1 \mathrm{~g}$ en las etapas de evaporación y concentración (mieles) y en la de punteo y batido (panela), para posteriormente ser pulverizadas en un molino con cuchillas extrafinas; consecutivamente se disolvieron en $100 \mathrm{~mL}$ de agua tipo HPLC y se centrifugaron a 4000 rpm durante 30 minutos, para retirar la mayor parte de sólidos presentes; cada muestra se inyectó al equipo por triplicado, posteriormente, se extraen los datos reportados por el equipo de las áreas bajo la curva, y de esta manera se interpoló en la ecuación de la recta, ecuación (1) y se determinó la concentración de analito presente en cada una de las muestras.

$$
y=b x+a
$$

Dónde:

$x$ : Concentración del analito.

$y$ : Relación entre el área de integración del señal correspondiente a la transición.

$a$ : el intercepto.

$b$ : es la pendiente.

Estandarización del método para la determinación de acrilamida por HPLC. La estandarización para el análisis de acrilamida se realizó en una columna C18 de 4,6 x $150 \mathrm{~mm}$, tamaño de partícula $5 \mu \mathrm{m}$, a una temperatura de $23^{\circ} \mathrm{C}$ y un flujo de $0,5 \mathrm{~mL} / \mathrm{min}$, con un detector UV-Vis a $210 \mathrm{~nm}$. El volumen de

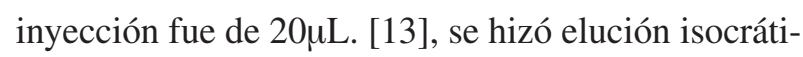
ca con Acetonitrilo:Agua, 1:24 (v/v). Para realizar la corrida cromatográfica, se inyectaron cada uno de los estándares de acrilamida y las muestras de panela (blanca, negra y granulada). El tiempo de corrida fue de 6 minutos para los estándares de acrilamida, debido a que después de este tiempo no aparecían más señales y 30 minutos para las muestras [13], para observar si aparecían nuevas señales durante este tiempo de corrida.

Se preparó una solución stock de acrilamida, 100 $\mathrm{mg} / \mathrm{kg}$ utilizando reactivo grado analítico, acrilamida electroforesis $99.9 \%$ Merck, esta disolución se preparó empleando un balón aforado clase A. Posteriormente se hicieron disoluciones a diferentes concentraciones de 0,$4 ; 1 ; 2 ; 5 ; \mathrm{y} 10 \mathrm{mg} / \mathrm{kg}$ respectivamente, con lo cual se obtuvo la curva de calibración para la cuantificación de acrilamida en las muestras.

\section{Resultados y discusión}

\subsection{Estandarización del método analítico}

Se evaluaron algunos de los parámetros básicos de una validación como linealidad, precisión, exactitud, límite de detección, límite de cuantificación y selectividad. La linealidad fue establecida a partir de disoluciones acuosas del stock de acrilamida, a los niveles de concentración de 0,4; 1,0; 2,0; 5,0 y $10 \mathrm{mg} / \mathrm{Kg}$., a lo largo de todo el proceso de estandarización realizando estas curvas por quintuplicado obteniendo un $r^{2}$ con un valor promedio de 0,99984 (figura 1). Los coeficientes de correlación obtenidos fueron muy cercanos a la unidad prevaleciendo una correlación positiva. Existiendo una dependencia entre las 2 variables, denominada relación directa: indicando que a medida que aumenta la concentración, aumenta el área. El criterio de aceptación para este método analítico es un coeficiente de correlación $\leq 0,95$ y por los datos obtenidos las linealidades se consideran válidas. A partir de la evaluación de las pendientes obtenidas en cada una de las rectas de regresión, se puede decir que el método con un mayor valor en su pendiente es más sensible, es decir que mayor es la respuesta del método frente a los cambios de concentración del analito.

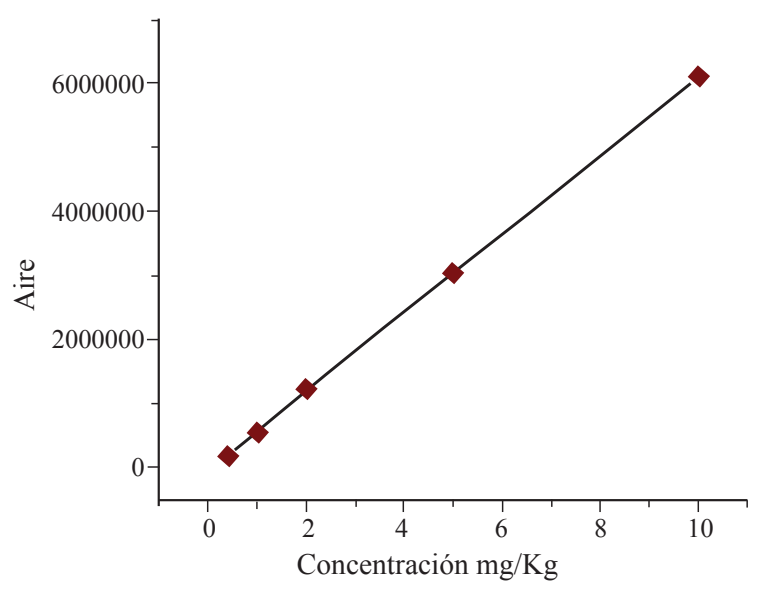

Figura 1. Curva de calibración de acrilamida.

La precisión del método se evalúo por medio de la repetitividad y la reproducibilidad. En el caso de repetitividad, el estudio se realizó por quintuplicado para cada muestra a una concentración de 0,4 , 1,0 y $10 \mathrm{mg} / \mathrm{kg}$. Esta valoración y las diluciones se prepararon y analizaron por un mismo analista, el mismo día. Y en el caso de la reproducibilidad, el estudio se realizó por quintuplicado para cada muestra, a una concentración de $1 \mathrm{mg} / \mathrm{kg}$. La valoración 
y las diluciones se prepararon por 3 analistas, en 3 días diferentes, obteniendo una diferencia porcentual RSD para cada uno de los analistas de 0,$5 ; 1,9$; y $1,1 \%$. El promedio obtenido en el\% RDS fue de 1,16 , lo cual proporciona una adecuada sensibilidad para desarrollar el método propuesto.

Para determinar la exactitud se obtuvo un porcentaje de recuperación de 101,4, y osciló con valores entre 92,2 y 106,0 , con un \% RSD entre 1,1 y 0,2 , para un promedio de 0,6 . La exactitud del método se determinó con 10 repeticiones a diferentes concentraciones.

Los límites de detección (LOD) del análisis cuantitativo indican el nivel más bajo del analito que puede medirse con certeza en una muestra, equivalente a tres veces la desviación estándar del ruido en análisis, mientras que el límite de cuantificación (LOQ) se calcula a partir de la concentración de los analitos que proporcionan señales iguales a diez veces la relación señal- ruido en el análisis. El LOD y el LOQ calculados fue de 0,0008 y $0,01 \mathrm{mg} / \mathrm{kg}$, respectivamente, satisfaciendo adecuadamente la cuantificación de acrilamida en panela por el método HPLC.

La selectividad se verificó al emplear un estándar certificado de acrilamida, el cual indica que el tiempo de retención es único para esta sustancia y que no se van a encontrar interferencias. Además, la resolución es mayor a 0,5 lo cual indica que no se van a encontrar dos señales en el mismo tiempo de retención.

\subsection{Determinación y cuantificación de acrilamida en panela}

Para identificar y cuantificar la presencia de acrilamida en las muestras seleccionadas (panela) se utilizó la curva de calibración (figura 1). Donde se interpolaron los valores de concentración del analito en cada una de las muestras, realizándolo por triplicado, a partir de las áreas reportadas por el equipo, se promediaron estos valores y se reportaron en la tabla 1. El cromatograma correspondiente se presenta en la figura 2.

A continuación se presentan las diferentes etapas de elaboración de la panela y el efecto de la temperatura y el tiempo, que son los factores de mayor trascendencia para la formación de acrilamida.
Tabla 1. Concentración de acrilamida para las muestras analizadas.

\begin{tabular}{lc}
\hline Panela & $\begin{array}{c}\text { Concentración de } \\
\text { acrilamida }(\mathrm{mg} / \mathrm{Kg})\end{array}$ \\
\hline Blanca & 2 \\
Negra & 1,8 \\
Granulada & 1,6 \\
Jugo de caña & 0,0 \\
\hline
\end{tabular}

Fuente: Autores

Tabla 2. Concentración de acrilamida en las diferentes etapas de elaboración de la panela.

\begin{tabular}{lccc}
\hline $\begin{array}{l}\text { Etapas para la } \\
\text { elaboración de } \\
\text { la panela }\end{array}$ & $\begin{array}{c}\text { Temperatura } \\
{ }^{\circ} \mathrm{C}\end{array}$ & $\begin{array}{c}\text { Concentración } \\
\text { de acrilamida/ } \\
\mathrm{mg} / \mathrm{kg}\end{array}$ & Áreas \\
\hline $\begin{array}{l}\text { Jugo de caña } \\
\text { apronte }\end{array}$ & Ambiente & $\mathrm{ND}$ & $\mathrm{ND}$ \\
$\begin{array}{l}\text { Jugo de caña } \\
\text { fase 2 } \\
\text { clarificación }\end{array}$ & 40 a 85 & $\mathrm{ND}$ & $\mathrm{ND}$ \\
$\begin{array}{l}\text { Jugo de caña } \\
\text { encalado }\end{array}$ & 75 a 95 & $\mathrm{ND}$ & $\mathrm{ND}$ \\
$\begin{array}{l}\text { Jugo de caña } \\
\text { concentración y } \\
\text { evaporación }\end{array}$ & 100 a 110 & 0,8 & 389,592 \\
$\begin{array}{l}\text { Miel punteo } \\
\text { Miel batido y } \\
\text { enfriamiento }\end{array}$ & 100 a 120 & 1.2 & 595,824 \\
Panela & 0 & 2,0 & 996,827 \\
& ND: & 2,2 & $1,076,543$ \\
\hline
\end{tabular}

Fuente: Autores

En la tabla 2 se puede observar la formación de acrilamida a partir de la etapa de concentración y evaporación,en la que se evidencia un aumento de temperatura sobre los $100{ }^{\circ} \mathrm{C}$; se puede deducir que la formación de acrilamida es directamente proporcional a la temperatura y al tiempo, y que la máxima concentración se alcanza en el producto final, con una concentración de $2,2 \mathrm{mg} / \mathrm{kg}$; esto lleva a ratificar el efecto de la reacción de Maillard, por sus principales precursores: la asparagina y los azucares reductores.

\section{Conclusiones}

El método desarrollado es simple,reproducible y permite evidenciar la presencia de acrilamida en las diferentes etapas de la producción de la panela; estableciéndose para la precisión un RDS de 0,5 a 1,0\%, y para la exactitud, un promedio de porcentaje de recuperación de $101,4 \%$, lo cual indica que el método es confiable. 


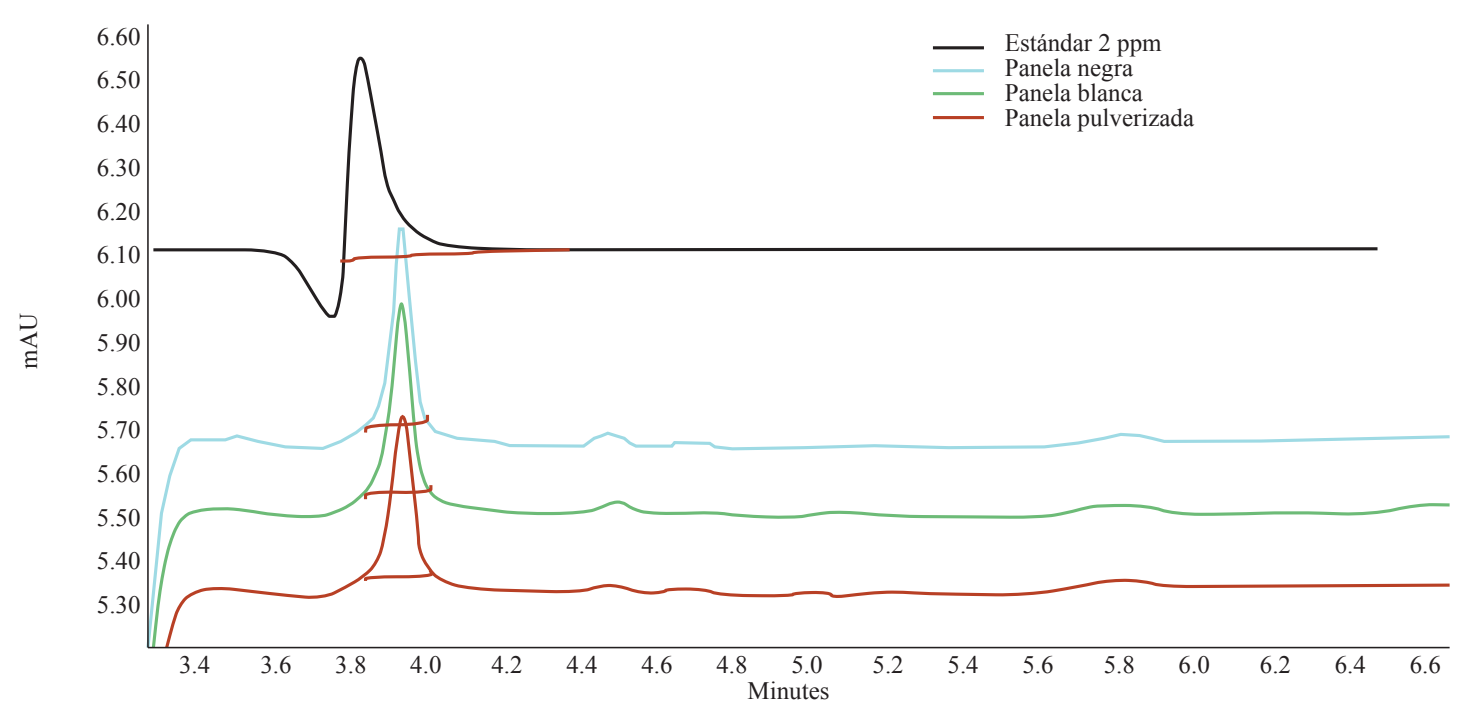

Figura 2. Muestras de panela con estándar $2 \mathrm{mg} / \mathrm{Kg}$.Fuente: autores

Se implementó y evaluó una técnica de HPLC, fase reversa mediante la cual se pudo detectar y cuantificar acrilamida en las siguientes etapas:concentración y evaporación, miel punteo, miel batido y enfriamiento de panela.

No se detectó acrilamida desde la obtención del jugo hasta la clarificación. A partir de la evaporación hasta el producto final, las concentraciones fueron entre $0,8-2,0 \mathrm{mg} / \mathrm{kg}$.

La temperatura constituye un parámetro físico muy importante, que incide en la formación de acrilamida en el proceso de elaboración de la panela, determinándose su formación acelerada a altas temperaturas.

Este trabajo constituye un primer paso en la estimación del riesgo por la generación de acrilamida en panela y en el análisis de las posibles medidas de gestión para aplicar en los trapiches paneleros, y así buscar reducir su presencia.

\section{Referencias}

[1] D. R. C. Lineback, James R Sadler, Richard H., "Acrylamide in foods: a review of the science and future considerations", Annual review of food science and technology, vol. 3, pp. 15-35, 2012.

[2] A. Claus, R. Carle, and A. Schieber, "Acrylamide in cereal products: A review", Journal of Cereal Science, vol. 47, pp. 118-133, 3, 2008.

[3] P. E. Boon, A. de Mul, H. van der Voet, G. van
Donkersgoed, M. Brette, and J. D. van Klaveren, "Calculations of dietary exposure to acrylamide", Mutation Research/Genetic Toxicology and Environmental Mutagenesis, vol. 580, pp. 143-155, 2005.

[4] M. Anese, M. Suman, and M. C. Nicoli, "Acrylamide removal from heated foods", Food Chemistry, vol. 119, pp. 791-794, 2010.

[5] D. A. Vattem and K. Shetty, "Acrylamide in food: a model for mechanism of formation and its reduction", Innovative Food Science $\mathcal{E}$ Emerging Technologies, vol. 4, pp. 331-338, 92003.

[6] G. Carrieri, M. Anese, B. Quarta, M. V. De Bonis, and G. Ruocco, "Evaluation of acrylamide formation in potatoes during deep-frying: The effect of operation and configuration", Journal of Food Engineering, vol. 98, pp. 141-149, 5 2010.

[7] R. C. Alves, C. Soares, S. Casal, J. O. Fernandes, and M. B. P. P. Oliveira, "Acrylamide in espresso coffee: Influence of species, roast degree and brew length", Food Chemistry, vol. 119, pp. 929-934, 2010.

[8] E. Tareke, P. Rydberg, P. Karlsson, S. Eriksson, and M. Törnqvist, "Analysis of Acrylamide, a Carcinogen Formed in Heated Foodstuffs", Journal of Agricultural and Food Chemistry, vol. 50, pp. 4998-5006, 2002. 
[9] G. R. Borray, "La Agroindustria Rural De La Panela En Colombia Roles, Problemática Y Nuevos Retos", Corpoica 2006.

[10] Agropecuaria., "Composición de panela en bloque de acuerdo con muestras tomadas en diferentes regiones del país. Análisis realizados en laboratorios de CORPOICA-CIMPA entre 1988 y 2004', Centro de investigación Tibaitata, 2004.
[11] UERIA, "Concepto cientifico acrilamida en panela", 2012.

[12] Alimentos, "Concepto cientifico Poliacrilamida en Panela", 2011.

[13] Luis Robinson Camelo Caballero, "Validación de una metodologia analitica para la determinacion y cuantificacion de acrilamida en hojuelas de platano maduro (musa paradisiaca) por la tecnica HPLC.", 2010. 